\title{
Non Symmetrical Flows in Liquid Metal Systems*
}

\author{
By Martha SALCUDEAN** and Roderick I. L. GUTHRIE***
}

\begin{abstract}
Synopsis
$A$ mathematical model of liquid flow in cylindrical vessels has been developed and applied to the case of ladle tapping operations. The results of the work indicate that significant three dimensional effects are to be expected when jets penetrate a bath of steel in an off centre andlor inclined mode, and these are described in the text. Comparisons with experimental flow fields demonstrate the predictive capability of the present model.
\end{abstract}

\section{Introduction}

There are many examples in liquid metal processing, where liquid is in motion. In such cases, continuous phases are in bulk motion and surface tension forces are not normally significant. Nevertheless, the flows are usually complex in character, often being transient, turbulent and three dimensional as a result of system geometries. As these flows have a considerable bearing on any heat and mass transfer processes occurring, it is important that process metallurgists develop a better understanding of the fluid flow phenomena associated with typical industrial systems.

\section{Mathematical Formulation}

\section{Problem Statement}

As the fluid dynamics involved are complex in nature, it is appropriate to develop stepwise approaches to the mathematical modelling of these systems. However, the general hydrodynamic equations describing such turbulent flows of liquid can still be represented, and these are written as follows using index notation:

Conservation of mass :

$$
\frac{\partial \rho}{\partial t}+\frac{\partial \rho u_{j}}{\partial x_{j}}=0
$$

Conservation of momentum:

$$
\frac{\partial \rho u_{i}}{\partial t}+\frac{\partial \rho u_{i} u_{j}}{\partial x_{j}}=-\frac{\partial p}{\partial x_{i}}+f_{i}+\frac{\partial}{\partial x_{j}}\left(\mu \frac{\partial u_{i}}{\partial x_{j}}-\rho u_{i}^{\prime} u_{j}^{\prime}\right)
$$

As liquids can be normally regarded as incompressible, the general continuity equation (1), then requires zero divergence of flow. Similarly, in the equation of motion (Eq. (2)) the first two terms then represent the substantial derivative of the velocity multiplied by constant density and expresses the spatial and time dependence of velocity $u_{i}$. These con- vective terms are balanced by pressure forces, body forces (e.g., gravitational, electromagnetic, centrifugal) and finally the molecular and turbulent viscous stresses.

\section{Turbulence Models}

In some instances, turbulence phenomena can be of prime importance (e.g., combustion processes, particle dispersions in melts) and modelling should be considered. In this regard, both algebraic and differential models of turbulence can be considered. The former are very attractive in terms of their simplicity and lower inherent computational costs in obtaining numerical solutions of flow problems. However, and particularly for recirculating flow systems, it is more appropriate to presume that turbulence characteristics (i.e., turbulence intensity and length scale) are not "local" properties but depend on events occurring elsewhere in the flow. In such circumstances a differential turbulence model provides a better representation of the flow than the simple algebraic models.

Since models based on a transport equation containing $l$ explicitly have not proved successful, it is customary to express the dissipation of turbulence in terms of alternative turbulence quantities containing $l$ implicitly. Thus, in the $k-\varepsilon$ model used, ${ }^{1)} l$ appears in the form of an energy dissipation term, $\varepsilon$, where

$$
\varepsilon=\frac{k^{3 / 2}}{l}
$$

so that the turbulent viscosity, $\mu_{t}^{\dagger}$, becomes

$$
\mu_{t}=\underset{\varepsilon}{C_{\mu} \rho k^{2}}
$$

The two differential equations describing the steady state transport of $k$ and $\varepsilon$ from one part of the flow system to the other are again written for convenience and the sake of clarity in index form below:

$$
\frac{D(\rho k)}{D t}=\frac{\partial}{\partial x_{j}}\left(\begin{array}{cc}
\mu_{\mathrm{t}} & \partial k \\
\sigma_{\mathrm{k}} & \partial x_{j}
\end{array}\right)+\mu_{\mathrm{t}} G-\rho \varepsilon
$$

and

$$
\frac{D(\rho \varepsilon)}{D t}=\frac{\partial}{\partial x_{j}}\left(\frac{\mu_{\mathrm{t}}}{\sigma_{\varepsilon}} \frac{\partial \varepsilon}{\partial x_{j}}\right)+\frac{C_{1} \varepsilon}{k} \mu_{\mathrm{t}} G-C_{2} \rho \frac{\varepsilon^{2}}{k}
$$

where,

* Received May 21, 1980.

** University of Ottawa, Ottawa, Ontario K1N 6N5, Canada.

*** McGill University, Montreal, Quebec, P.Q. H3A 2A7, Canada.

$\uparrow \quad$ The Prandtl-Kolmogorov formula for $\mu_{t}$ applies; $\mu_{t}=p l k^{1 / 2}$. 


$$
G \equiv\left(\frac{\partial u_{i}}{\partial x_{j}}+\frac{\partial u_{j}}{\partial x_{i}}\right) \frac{\partial u_{i}}{\partial x_{j}} .
$$

The right side of Eq. (5) contains the diffusion, generation and dissipation terms for $k$. Similarly Eq. (6) expresses the fact that changes in $\varepsilon$ along a streamline arise through the influence of diffusion transport through interactions of the turbulence with the mean flow and through self interactions of the turbulence. The values of the various constants appearing in these equations and used in the numerical computations are given in Table 2 of Ref. 2) where Eqs. (5) and (6) were presented in cylindrical form.

\section{Basic Equations for 3D Flow in Vertical Cylinders}

In the present work, the case of three dimensional flow phenomena in cylindrical containers are analysed, in view of its general relevance to metallurgical processes. For instance, inclined jets entering cylindrical vessels, centre or off centre, occur in such diverse systems as ladle tapping operations in steelmaking ${ }^{3)}$ or in the separation of alumina trihydroxide in the Bayer Process. ${ }^{4)}$ A transient laminar flow model, based on the MAC Los Alomos method, was developed for this work.

In view of the authors' previous research on furnace/ladle tapping operations in steelmaking, the specific vessel chosen for presentation of the present fluid flow model was a typical full scale teeming ladle of $2.24 \mathrm{~m}$ diameter. Consequently, various jet positions and angles of inclination were considered, for a typical ladle filling time of $210 \mathrm{~s}$.

Considering the case illustrated (see Fig. 1.) showing the entry of a non-vertical jet into a cylindrical ladle, the relevant cylindrical form of the partial differential equations requiring solutions are presented as follows:

Continuity equation:

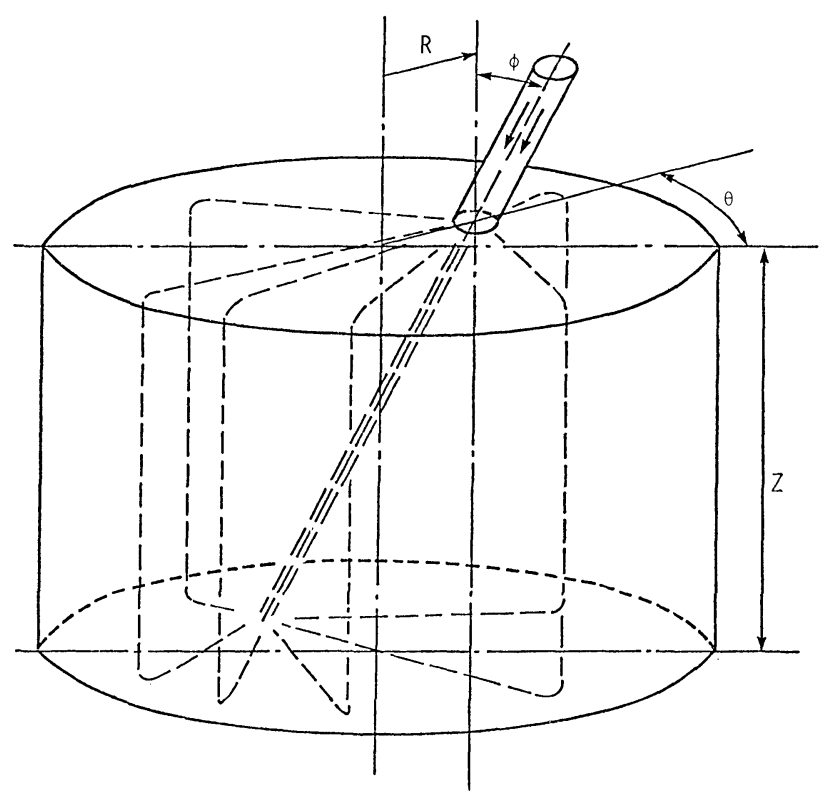

Fig. 1 (a). Off-centred inclined jet flowing into filling ladle.

$$
\frac{\partial u}{\partial R}+\frac{1}{R} \frac{\partial v}{\partial \theta}+\frac{\partial w}{\partial z}+\frac{u}{R}=0
$$

The three momentum equations for incompressible laminar flow are written as follows:

$$
\begin{aligned}
& \frac{\partial u}{\partial t}+\frac{\partial u^{2}}{\partial R}+\frac{1}{R} \frac{\partial(u v)}{\partial \theta}+\frac{\partial(u w)}{\partial z}+\frac{u^{2}}{R}-\frac{v^{2}}{R} \\
& =G R-\frac{1}{\rho} \frac{\partial p}{\partial R}+\nu\left(\frac{\partial^{2} u}{\partial R^{2}}+\frac{1}{R} \frac{\partial u}{\partial R}+\frac{1}{R^{2}} \frac{\partial^{2} u}{\partial \theta^{2}}\right. \\
& \left.+\frac{\partial^{2} u}{\partial z^{2}}-\frac{2}{R^{2}} \frac{\partial v}{\partial \theta}-\frac{u}{R^{2}}\right) \\
& \frac{\partial v}{\partial t}+\frac{\partial(u v)}{\partial R}+\frac{1}{R} \frac{\partial\left(v^{2}\right)}{\partial \theta}+\frac{\partial(w v)}{\partial z}+\frac{2}{R} v u \\
& =G \theta-\frac{1}{\rho R} \frac{\partial p}{\partial \theta}+\nu\left[\frac{\partial^{2} v}{\partial R^{2}}+\frac{1}{R} \frac{\partial v}{\partial R}+\frac{1}{R^{2}} \frac{\partial^{2} v}{\partial \theta^{2}}\right. \\
& \left.+\frac{\partial^{2} v}{\partial z^{2}}+\frac{2}{R^{2}} \frac{\partial u}{\partial \theta}-\frac{v}{R^{2}}\right] \\
& \frac{\partial w}{\partial t}+\frac{\partial(w u)}{\partial R}+\frac{1}{R} \frac{\partial(w v)}{\partial \theta}+\frac{\partial w^{2}}{\partial z}+\frac{u w}{R} \\
& =G Z-\frac{1}{\rho} \frac{\partial p}{\partial z}+\nu\left[\frac{\partial^{2} w}{\partial R^{2}}+\frac{1}{R} \frac{\partial w}{\partial R}+\frac{1}{R^{2}} \frac{\partial^{2} w}{\partial \theta^{2}}\right. \\
& \left.+\frac{\partial^{2} w}{\partial z^{2}}\right]
\end{aligned}
$$

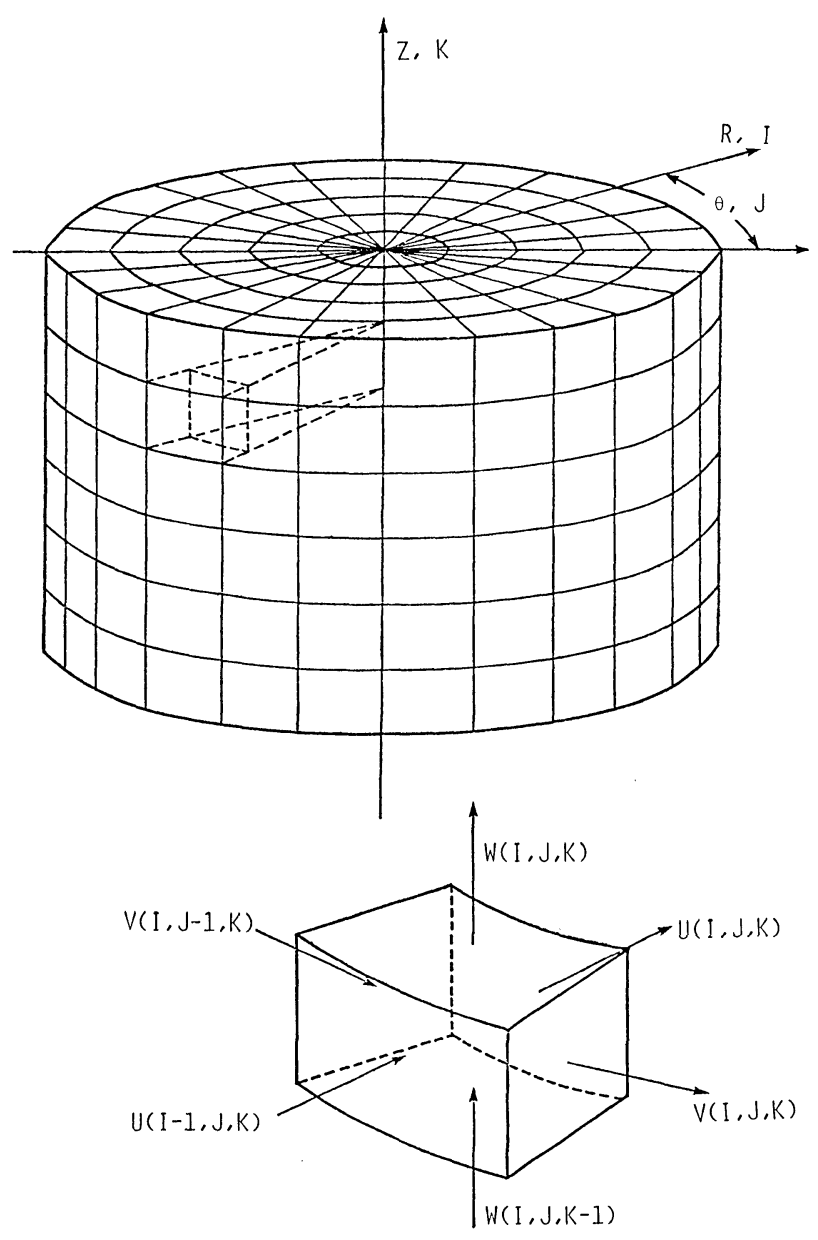

Fig. 1 (b). Construction of finite difference grid. 
The following set of boundary conditions were applied:

$$
\begin{aligned}
& R=R_{i} \quad w=W_{j} \cos \phi \\
& u=W_{j} \sin \phi \\
& -W_{j} R \Delta R \Delta \theta=\frac{d}{d t} \int_{0}^{R_{\mathrm{L}}} \int_{0}^{2 \pi} H_{\mathrm{t}, \mathrm{R}, \theta} R d R d \theta \\
& 0<t<T \quad z=H_{\mathrm{t}, \mathrm{R}, \theta}, P=P_{\mathrm{a}}, v=m_{j} / \rho \pi R_{\mathrm{L}}^{2} ; \\
& \frac{\partial u}{\partial z}=\frac{\partial w}{\partial z}=0 \\
& 0<t<T\left\{\begin{array}{l}
0<R<R_{\mathrm{I}} \\
z=0 \\
0<\theta<2 \pi
\end{array}\right. \\
& 0<t<T\left\{\begin{array}{l}
0<z<H \\
R=R_{\mathrm{L}} \\
0<\theta<2 \pi
\end{array} \quad v=0, w=0, u=0 \ldots \ldots[\mathrm{BC}-5]\right.
\end{aligned}
$$

The first condition considers that a coherent tapping stream enters the ladle with a uniform velocity $W_{j}$, at an angle $\phi$, and mass flow, $m_{j}$, to the vertical. The second boundary condition requires conservation of mass, stating that the volume of jet inflow per unit time is equal to the rate of increase in the total volume of liquid within the vessel.

The third condition refers to the rising surface of the bath and requires the pressure to be uniform (i.e., ambient atmospheric pressure) as well as the divergence of liquid to be zero, and zero shear stresses at the free surface.

The remaining boundary conditions specify nonslip conditions at the impermeable walls of the ladle.

\section{Numerical Procedures}

Finite difference techniques were adopted to solve the system of differential equations: twenty eight radial, and ten angular nodes were considered. A typical control volume is shown in Fig. 1 (b) together with velocities at the sides of the cell and pressure in the cell centre.
Artificially high viscosities of $10^{2} \sim 10^{3}$ molecular values were used as a first approximation of turbulent simulation. ${ }^{2)}$

An upwind differential explicit method was used. Equivalent finite difference expressions for the mass conservation and momentum equations are given in the Appendix. A Gourant type of condition for stability had to be satisfied such that:

$$
\Delta t<(0.2-0.5)\left|\frac{\Delta x_{i}}{u_{i}}\right|
$$

\section{Computed Results and Discussion}

To test the reliability of the three dimensional model, computations were first performed for the case of a central vertical jet and flow fields compared with those predicted on the basis of a two dimensional axisymmetric model developed previously by the authors. $^{3)}$

\section{Case A (Centred Jet)}

Figure 2 illustrates the predicted flow in the ladle for such an axisymmetrical jet. As expected the present model correctly shows the flow in the vessel to be symmetrical and agrees precisely with previous computations.

High velocities are to be observed in the region adjacent to the jet, while the velocities along the bottom of the ladle decrease from the centre out to the wall.

The vortex centre is seen to be located much closer to the wall than to the axis of symmetry, and one observes that the velocities are technologically significant, being of the same order of magnitude as rising velocities of buoyant alloy additions (i.e. typical lump additions of aluminum, ferro-silicon, silico-manganese). ${ }^{5)}$

\section{Case B (Inclined Jet)}

The influence of jet inclination is next presented, in Fig. 3, where the flow pattern for a central jet

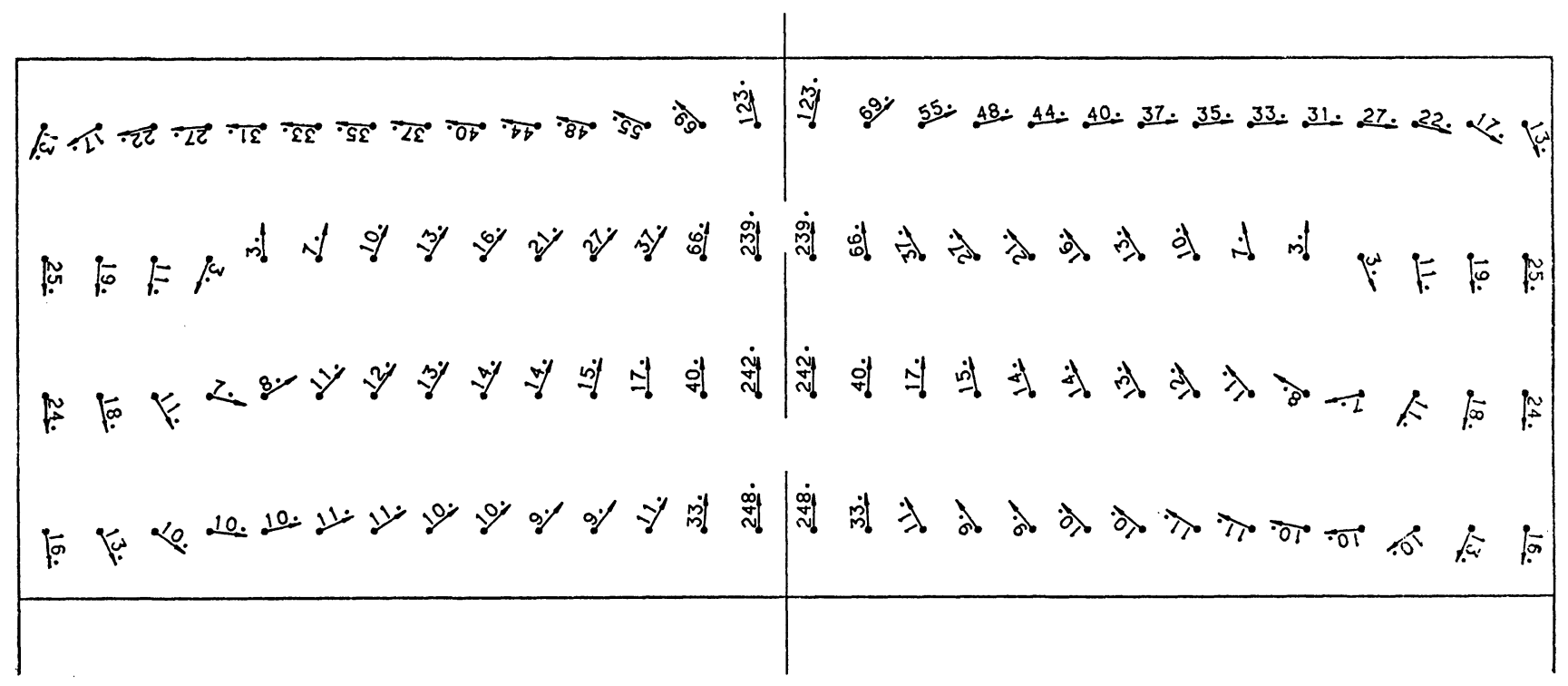

Fig. 2. Flow pattern in cylindrical vessel for axisymmetrical jet. 
penetrating the bath at an angle, $\phi$, of $45 \mathrm{deg}$ to the vertical, is illustrated. One observes the jet maintaining its direction as it penetrates toward the bottom of the ladle. As expected, the high velocity regions move to coincide with the inclination of the penetrating jet. Since the jet is inclined from the right to the left, the rising velocities close to the left wall of the ladle are predicted to be considerably higher than those at the right wall in accord with experiment. $\left.{ }^{6}\right)$ Further, the left side of the Hills type vortex is further displaced towards the outer wall, while the right side moves towards the centre of the ladle. It is worth noting that much more turbulence can be expected to be generated in the left half of the vessel, leading to good mixing and dispersion of alloy addition material.

When comparing the difference in flows between Figs. 2 and 3, the need for three dimensional treatment (such as modelled here) is very evident. This becomes even more true once one has to treat offcentre inclined jets.

\section{Case C (Off-centred Jet)}

The off-centred vertical jet is illustrated in Fig. 4 where a vertical jet penetrates at the mid-distance between the axis of symmetry and the wall. Pronounced non-symmetry effects are predicted with considerably higher velocities in that half of the cylinder containing the jet as compared to the other. Similarly, the displacement of the left vortex centre towards the wall is again in evidence whereas the opposite displacement occurs in the other half of the cylindrical vessel. Tangential velocities are significant. These are shown in Fig. 4, where the numbers written below the arrows represent fluid velocity components $\left(\mathrm{cm} \cdot \mathrm{s}^{-1}\right)$ in the tangential direction. In the notation used, positive tangential components indicate

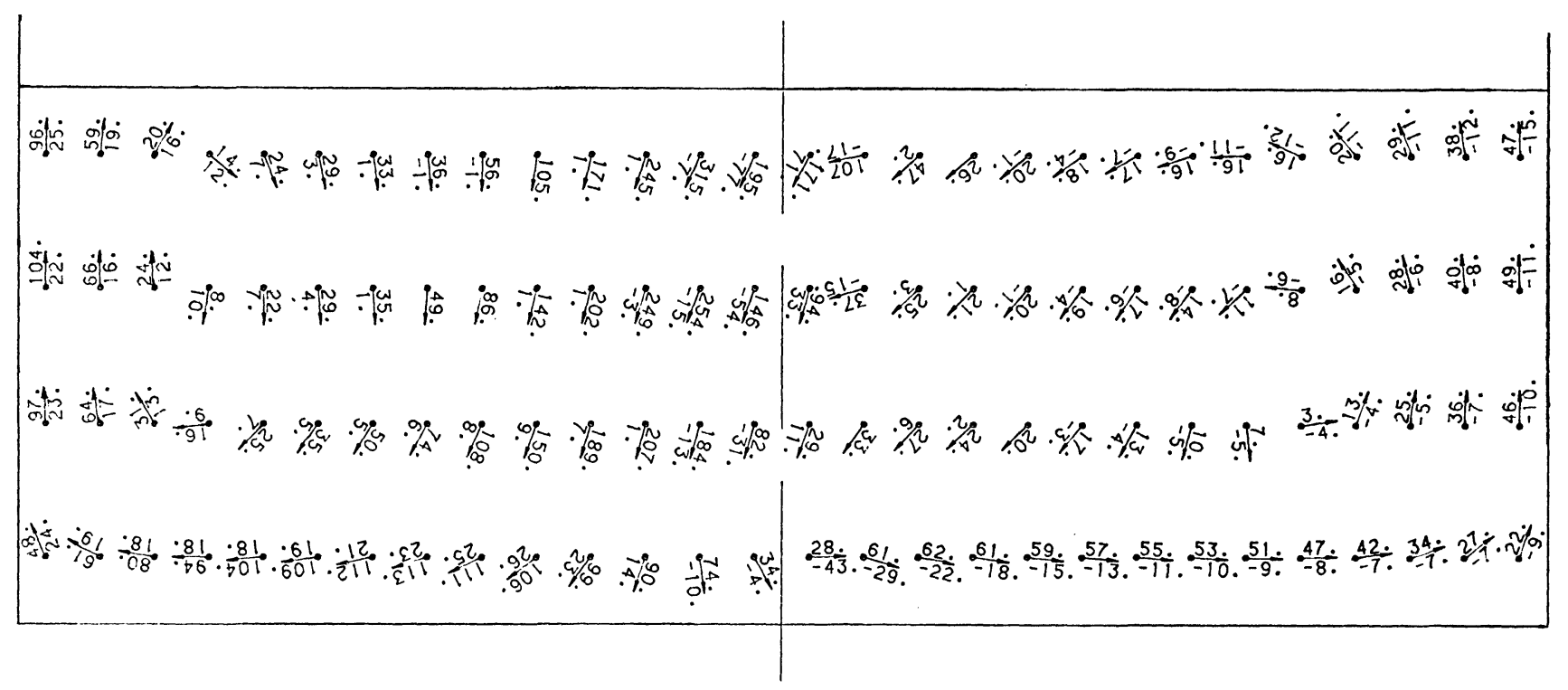

Fig. 3. Flow pattern in cylindrical vessel for central inclined jet.

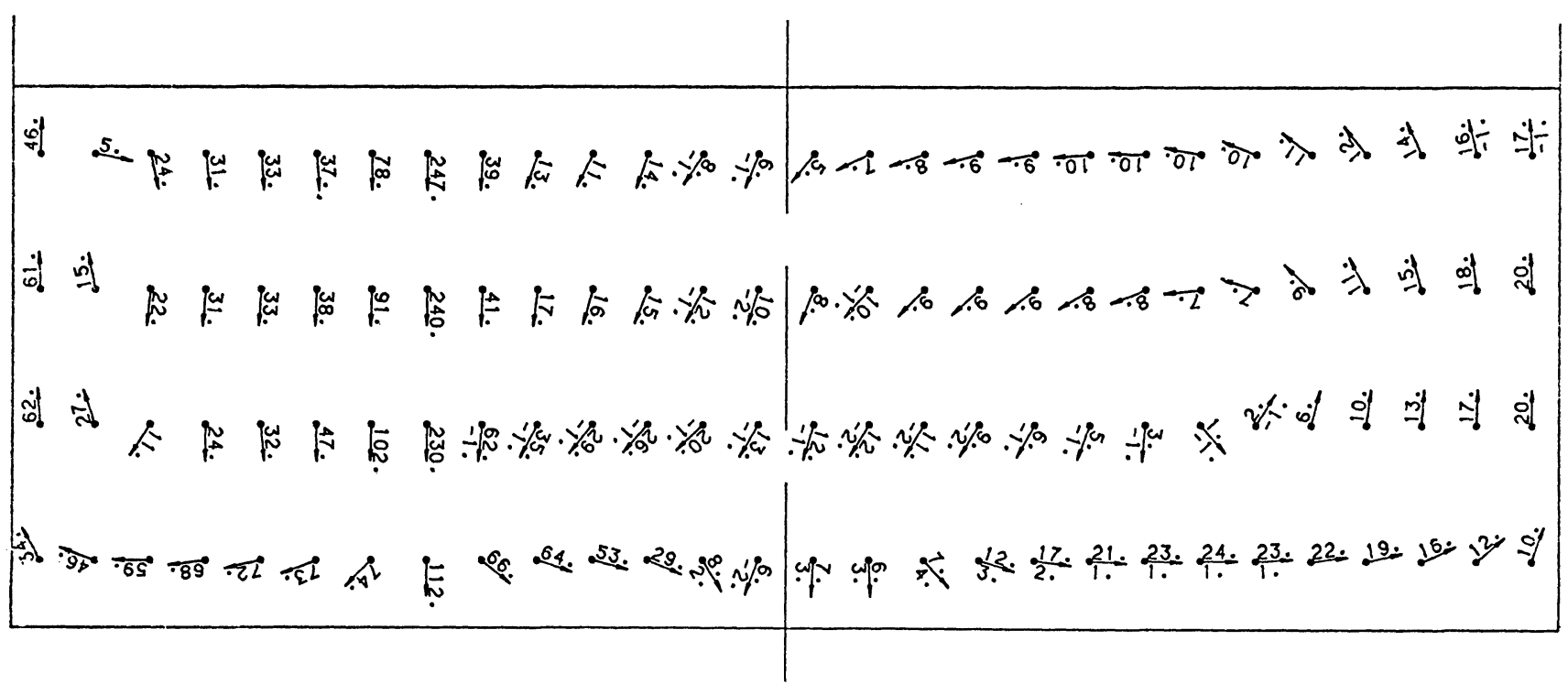

Fig. 4. Flow pattern in cylindrical vessel for off-centred vertical jet. 
counter clockwise directions, while negative components indicate clock-wise flow for an observer looking down on the filling bath. Referring to Fig. 4 once more, significant components are predicted and these arise as a result of the jet's inclination.

Figure 5 (a) illustrates the flow patterns predicted in the plane of the jet for an off-centred, inclined jet. Flow reversal can now be observed at the bottom of the ladle. This is caused by high horizontal velocity components of liquid entrained on the underside of the jet. Figure 5 (b) predicts the formation of secondary vortices. High tangential components are also to be observed.

\section{Comparison with Experimental Work}

In order to demonstrate the general validity of the model, the experimental flow patterns and velocity fields obtained by M. Tanaka using a $61 \mathrm{~cm}$ diameter transparent water model can be used as a base of reference. ${ }^{6}$ )

Thus, Figs. 6 (a) and (b) provide experimental and predicted flows for a vertical jet entering the cylindrical vessel at the mid-radius. Table 1 gives the physical parameters used for this study.

Somewhat more entrainment of fluid on the underside of the jet was observed in the water modelling work by $\mathrm{M}$. Tanaka. This was a consequence of higher local turbulence viscosities being generated in the jet boundary layer than that modelled. As a consequence, the velocity vectors in that region do not correspond entirely (see Figs. 6 (a) and (b)).

In other regions of the flow field however, very reasonable correspondence between velocity vectors (magnitude and direction) is to be observed.

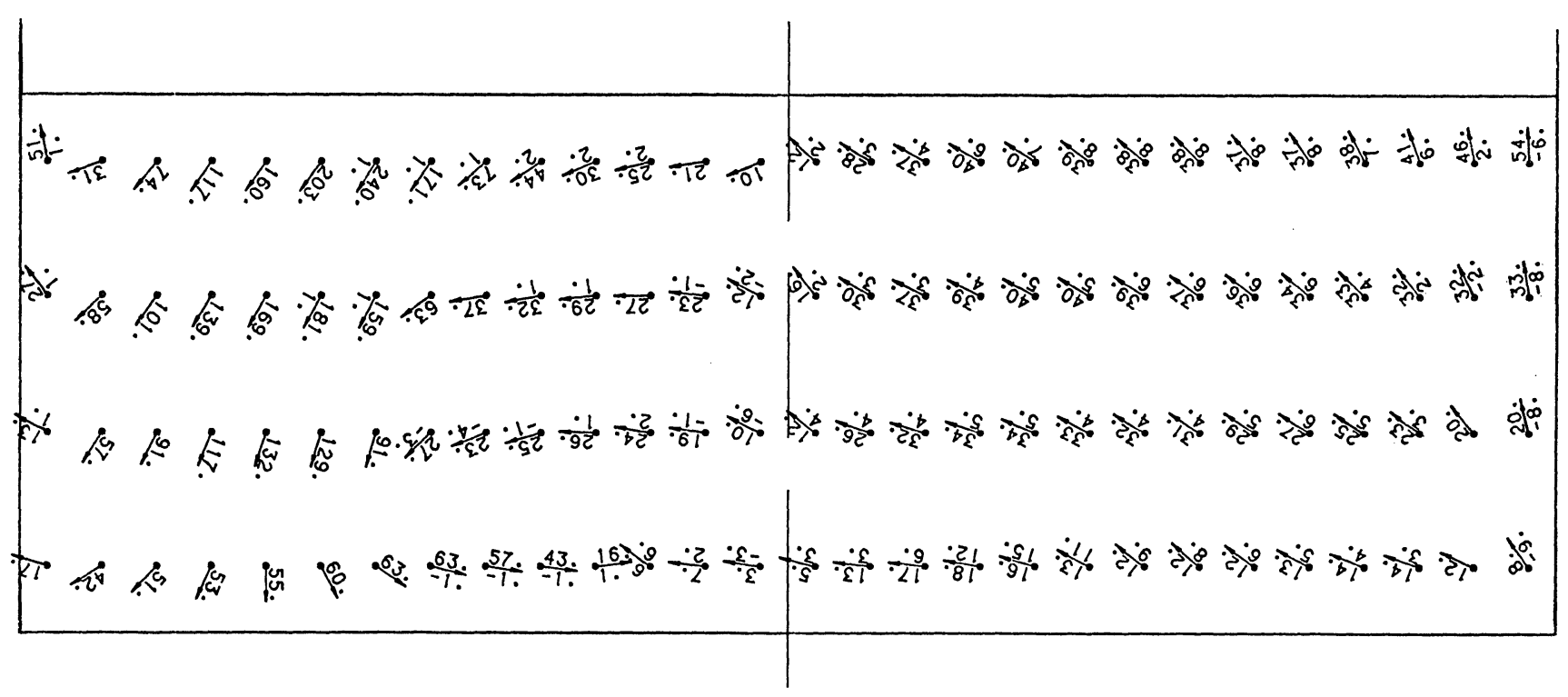

Fig. 5 (a). Flow pattern in cylindrical vessel for off-centred inclined jet. Plane of the jet.

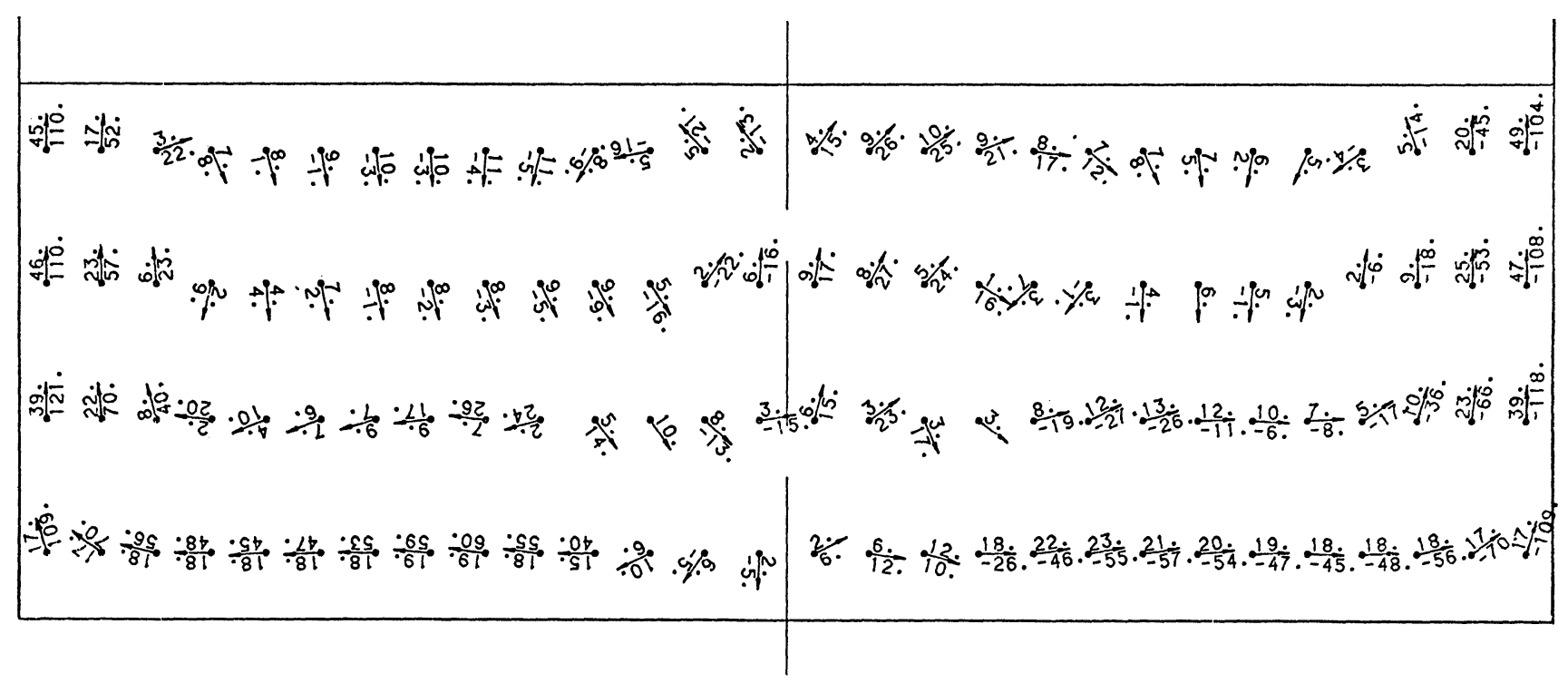

Fig. 5 (b). Flow pattern in cylindrical vessel for off-centred inclined jet. Plane perpendicular to the jet plane. 


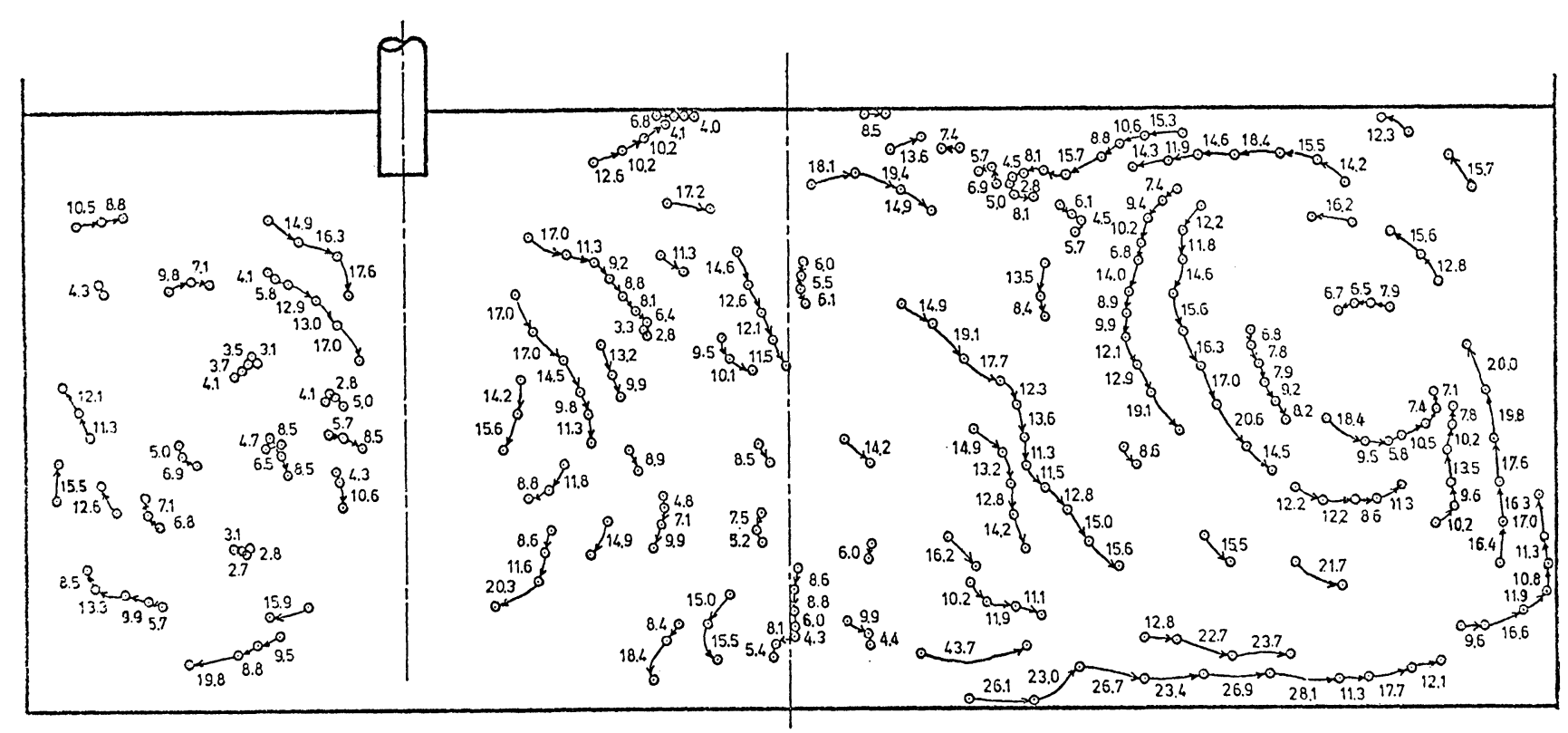

Fig. 6 (a). Flow pattern resulted from experimental data.

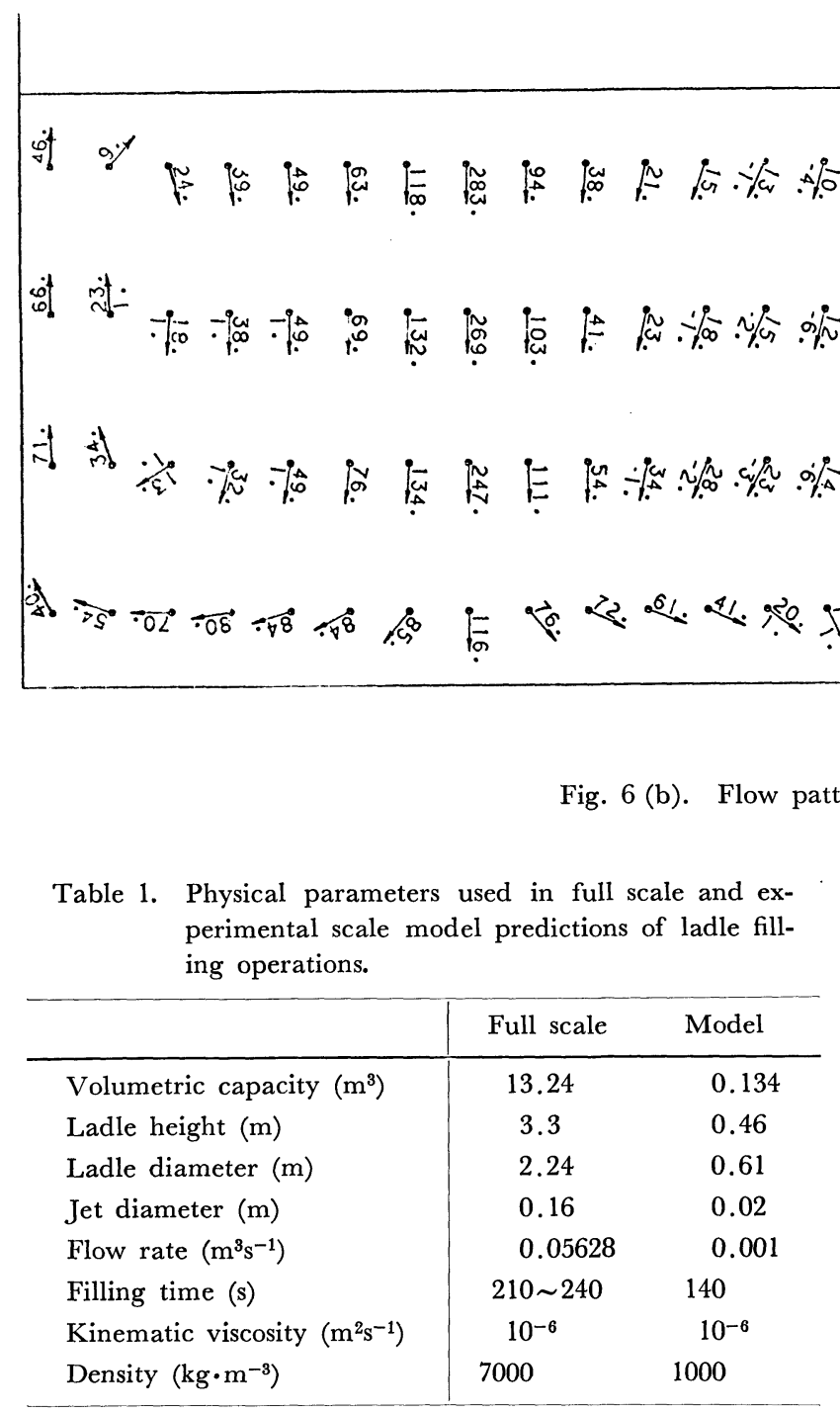

\section{Conclusions}

This work serves to illustrate the general capability of a three dimensional mathematical model of fluid flow. The specific computations chosen for presentation show that flow patterns generated in relatively complex flow systems can be predicted from first principles.

Since the full scale experimental modelling of metallurgical processing vessels can be prohibitively expensive as a result of their massive size, and since water modelling, at least of two phase flow systems, can, at best, be described as qualitative, ${ }^{7)}$ the use of three dimensional flow models in process metallurgical research and development work should become increasingly commonplace.

Further improvement on these predictive models is still needed. One area of importance for the specific case of steel jets plunging into ladles or continuous casting moulds, is the phenomenon of air entrain- 
ment. ${ }^{3)}$ This can lead to a complete reversal of flow in the upper regions of a filling ladle, and represents a fruitful area for further modelling efforts.

\section{Nomenclature}

$C$ : Constant

$D$ : Divergence

$f:$ Body force (gravitational, electromagnetic, etc.)

$G:$ Defined as $\left(\frac{\partial u_{i}}{\partial x_{j}}+\frac{\partial u_{j}}{\partial x_{i}}\right) \frac{\partial u_{i}}{\partial x_{j}}$

$G R, G \theta, G Z: \quad$ Body forces in $R, \theta, Z$ directions

$H$ : Height of bath

$k$ : Turbulence kinetic energy term

$l$ : Length scale

$m_{j}$ : Mass flow of jet

$p$ : Pressure

$P: \quad$ Pressure in finite difference equation

$R:$ Radius

$t$ : Time

T: Total tapping time

$u$ : Radial velocity

$v$ : Tangential velocity

$w:$ Vertical velocity

$W$ : Vertical velocity in finite difference form

$W_{j}$ : Velocity of penetrating jet

$x$ : General coordinate

$z$ : Coordinate

$\Delta:$ Increment

$\varepsilon:$ Energy dissipation

$\theta$ : Angular coordinate

$\mu$ : Dynamic viscosity

$\nu:$ Kinematic viscosity

$\rho$ : Density

$\sigma:$ Turbulence constant

$\phi:$ Jet inclination

\section{Superscript}

': Fluctuating component

\section{Subscripts}

a: Atmospheric

$i$ : Jet radius

$j$ : Jet

$\mathrm{k}$ : Turbulence

L: Ladle radius

$\mathrm{R}$ : Radial

t: Time

z: Coordinate

$\varepsilon$ : Energy dissipation

$\theta:$ Angular

$\mu:$ Index in constant

1: Constant in turbulence model

2: Constant in turbulence model

\section{Acknowledgements}

The authors wish to acknowledge Mr. A. Hurda and Mr. M. Rahman for their help and the National Research Council of Canada for their financial support.

\section{REFERENCES}

1) B. E. Launder, A. D. Gosman and J.H. Whitelaw: " Flow, Heat and Mass Transfer in Turbulent Recirculating Flows-Predictions and Measurements", Lecture notes, McGill University, Montreal, (1976).

2) M. Salcudean and R.I.L. Guthrie: Met. Trans., 9B (1978), 673.

3) M. Salcudean and R.I.L. Guthrie: Met. Trans., 9B (1978), 181.

4) J. L. Bray: Non-Ferrous Production Metallurgy, 2nd Ed., John Wiley \& Sons, New York, (1953).

5) H. Henein: M. E. Thesis, McGill University, (1975).

6) M. Tanaka: Ph.D. Thesis, McGill University, (1979).

\section{Appendix}

The following text provides a brief description of the numerical form of the continuity and momentum equations constructed for describing laminar three dimensional flow systems in cylindrical co-ordinates. Referring to the cell type shown in Fig. 1 (b), finite difference equations can be written using up-wind differencing as follows:

For the mass conservation equation,

$$
\begin{aligned}
D= & \frac{U(I, J, K)-U(I-1, J, K)}{\Delta R} \\
& +\frac{W(I, J, \kappa)-W(I, J, \kappa-1)}{\Delta Z} \\
& +\frac{V(I, J, K)-V(I, J-1, \kappa)}{(I-1.5) \Delta R \Delta \theta} \\
& +\frac{U(I, J, K)+U(I-1, J, K)}{2 \cdot \Delta R \cdot(I-1.5)}
\end{aligned}
$$

The momentum equations were written as follows for the radial direction:

$$
\begin{aligned}
& U(I, J, K)=U \mathcal{N}(I, J, K)+\Delta t[(P(I, J, K) \\
& \quad-P(I+1, J, K)) / \Delta R+G R-F U X-F U Y \\
& -F U Z-F U C+V I S R]
\end{aligned}
$$

Tangential component:

$$
\begin{gathered}
V(I, J, K)=V \mathcal{N}(I, J, K)+\Delta t[(P(I, J, K) \\
-P(I, J+1, K)) /((I-1.5) \cdot \Delta R \Delta \theta)+G \theta \\
-F V X-F V Y-F V C-F V Z+V I S \theta]
\end{gathered}
$$

Vertical component:

$$
\begin{aligned}
& W(I, J, K)=W \mathcal{N}(I, J, K)+\Delta t[(P(I, J, K) \\
& \quad-P(I, J, K+1)) / \Delta z+G Z-F W X-F W Y \\
& -F W Z-F W C+V I S Z]
\end{aligned}
$$

As an example the expressions are written for the momentum equation in the " $R$ " direction:

$$
\begin{aligned}
F U X= & \left\{[U \mathcal{N}(I, J, K)+U \mathcal{N}(I+1, J, K)]^{2}\right. \\
& \left.-[U \mathcal{N}(I-1, J, K)+U \mathcal{N}(I, J, K)]^{2}\right\} / 4 \Delta R \\
F U Y= & \{[U \mathcal{N}(I, J, K)+U \mathcal{N}(I, J+1, K)] \\
& \times[V \mathcal{N}(I, J, K)+V \mathcal{N}(I+1, J, K)] \\
& -[U \mathcal{N}(I, J-1, K)+U \mathcal{N}(I, J, K)] \\
& \times[V \mathcal{N}(I, J-1, K) \\
& +V \mathcal{N}(I+1, J-1, K)]\} /[4 \cdot \Delta \theta \cdot \Delta R(I-1.5)]
\end{aligned}
$$




$$
\begin{aligned}
F U Z= & \{[W \mathcal{N}(I, J, K)+W \mathcal{N}(I+1, J, K)] \\
& \times[U \mathcal{N}(I, J, K)+U \mathcal{N}(I, J, K+1)] \\
& -[W \mathcal{N}(I, J, K-1)+W \mathcal{N}(I+1, J, \kappa-1)] \\
& \times[U \mathcal{N}(I, J, K-1)+U \mathcal{N}(I, J, K)]\} / 4 \Delta Z \\
F U C= & \{[U \mathcal{N}(I, J, K)+U \mathcal{N}(I+1, J, K)] \\
& \times[U \mathcal{N}(I, J, K)+U \mathcal{N}(I+1, J, K)] \\
& +[U \mathcal{N}(I-1, J, K)+U \mathcal{N}(I, J, K)] \\
& \times[U \mathcal{N}(I-1, J, K) \\
& +U \mathcal{N}(I, J, K)]\} /[8(I-1) \Delta R]-\{[V \mathcal{N}(I, J, K) \\
& +V \mathcal{N}(I, J+1, K)]^{2}+[V \mathcal{N}(I, J, K) \\
& +V \mathcal{N}(I, J-1, K)]]^{2} /[8 \cdot \Delta R(I-1.5)] \\
V I S R= & 2[U \mathcal{N}(I+1, J, K)+U \mathcal{N}(I-1, J, K) \\
& -2 U \mathcal{N}(I, J, K)] / \Delta R^{2}+[U \mathcal{N}(I, J+1, K) \\
& +U \mathcal{N}(I, J-1, K) \\
& -2 U \mathcal{N}(I, J, K)] /\left[\Delta \theta^{2} \Delta R^{2}(I-1)^{2}\right] \\
& +[U \mathcal{N}(I, J, K+1)+U \mathcal{N}(I, J, K-1) \\
& -2 U \mathcal{N}(I, J, K)] / \Delta Z^{2}+[U \mathcal{N}(I+1, J, K) \\
& -U \mathcal{N}(I-1, J, K)] /\left[2 \Delta R^{2}(I-1)\right]
\end{aligned}
$$

$$
\begin{aligned}
& -U \mathcal{N}(I, J, K) /[(I-1) \Delta R]^{2}-[V \mathcal{N}(I, J+1, K) \\
& -V \mathcal{N}(I, J-1, K)] /\left[\Delta R^{2}(I-1.5)^{2} \Delta \theta\right]
\end{aligned}
$$

\section{Notation}

FUC, FUX, FUY, FUZ: Convective term in " $\mathrm{U}$ " momentum equation

$F V C, F V X, F V Y, F V Z$ : Convective terms in "V" momentum equation

$F W G, F W X, F W Y, F W Z$ : Convective terms in " $\mathrm{W}$ " momentum equation

$I, J, K:$ Gurrent mesh points coordinates

$\mathrm{U}:$ Radial velocity in finite difference equation

$U \mathcal{N}:$ Radial velocity in finite difference equation resulting from previous cycle.

VISR, VIS $\theta$, VISZ: Viscous terms in $R, \theta, Z$ directions

$V \mathcal{N}$ : Tangential velocity in finite difference equation resulting from previous cycle

V: Tangential velocity in finite difference equation ( $\theta$ direction)

$W: \quad$ Vertical velocity in finite diffrence equation

$W \mathcal{N}:$ Vertical velocity in finite difference equation resulting from previous cycle 